\title{
Cognitive vulnerability to depression: A comparison of the weakest link, keystone and additive models
}

\author{
Laura C. Reilly ${ }^{1}$, Jeffrey A. Ciesla ${ }^{1}$, Julia W. Felton ${ }^{2}$, Amy S. Weitlauf ${ }^{3}$, and Nicholas L. \\ Anderson ${ }^{1}$ \\ ${ }^{1}$ Psychology Department, Kent State University, Kent, OH, USA \\ 2Department of Psychology and Human Development, Vanderbilt University, Nashville, TN, USA \\ ${ }^{3}$ Department of Psychology, University of North Carolina, Chapel Hill, NC, USA
}

\begin{abstract}
Multiple theories of cognitive vulnerability to depression have been proposed, each focusing on different aspects of negative cognition and utilising different measures of risk. Various methods of integrating such multiple indices of risk have been examined in the literature, and each demonstrates some promise. Yet little is known about the interrelations among these methods, or their incremental validity in predicting changes in depression. The present study compared three integrative models of cognitive vulnerability: the additive, weakest link, and keystone models. Support was found for each model as predictive of depression over time, but only the weakest link model demonstrated incremental utility in predicting changes in depression over the other models. We also explore the correlation between these models and each model's unique contribution to predicting onset of depressive symptoms.
\end{abstract}

\section{Keywords}

Depression; Cognitive vulnerability; Weakest link

\begin{abstract}
Several models of cognitive vulnerability to depression have been proposed, asserting that individuals' cognitive characteristics confer varying degrees of susceptibility to depression (e.g., Abramson, Metalsky, \& Alloy, 1989; Beck, 1967; Brown \& Harris, 1978). These models differ in terms of the specific cognitions involved in this vulnerability, though the overarching concept is similar. That is, the presence of maladaptive beliefs places an individual at risk for depression, particularly in times of high stress. This common focus on particular cognitive content or beliefs differentiates these theories of depression from others that focus on maladaptive processes such as emotion regulation or depressive rumination (see Ciesla \& Roberts, 2007). As multiple forms of negative beliefs derived from these content-based theories have demonstrated promise in the prediction of depression, it is important for researchers to examine the interrelations among their respective
\end{abstract}

C 2012 Psychology Press, an imprint of the Taylor \& Francis Group, an Informa business Correspondence should be addressed to: Jeffrey A. Cielsa, Kent State University, 323 Kent Hall, Kent, OH 44242, USA. jciesla@kent.edu. 
vulnerabilities. Recently, researchers have speculated that risk for depression may vary as a function of an individual's greatest (or least) vulnerability.

Abela and Sarin (2002) proposed an idiographic approach to defining cognitive vulnerability. This new concept, termed the weakest link hypothesis, examines the influence of multiple measures of cognitive vulnerability such that a person's most maladaptive score on a set of indices is the best predictor of risk for depression. In a similar fashion, Morris, Ciesla, and Garber (2008) examined a keystone hypothesis in which a person's least maladaptive score on a set of cognitive measures is the best indicator of resiliency from depression. These two hypotheses, which consider the within-subject rank order of various cognitive indices to select individuals' greatest vulnerability or strength, have fared well in a handful of studies. Yet further research is needed to examine these hypotheses, and to test their validity compared to more parsimonious models of cognitive vulnerability. We first briefly introduce three major types of cognitive vulnerability implicated in depression, followed by an introduction to the literature on integrative models of these vulnerabilities, and then discuss the need for investigations that directly contrast different integrative models.

\section{Cognitive vulnerability to depression}

Perhaps the most widely known cognitive theory of psychopathology is Beck's cognitive theory of depression (Beck, 1967). Beck's theory posits that negative experiences in childhood may influence the development of enduring dysfunctional beliefs and cognitive schemas, which ultimately place individuals at risk for depression. Specifically, Beck described beliefs regarding the self, one's personal world, and the future as the negative cognitive triad (e.g., "If I don't succeed, I am a failure"). When such cognitive schemas are employed, individuals construct representations of reality that are consistent with symptoms of psychopathology. Since its development in 1967, tests of Beck's cognitive theory have largely been supported (see Beck, 2005; Ingram, Miranda, \& Segal, 1998, for reviews). More recently, theorists have offered alternative cognitive models emphasising the reactivity of some individuals' cognition. (Beck, 2005; Teasdale, 1988) Although many variations on these models have been proposed, they commonly present negative cognitive beliefs as key ingredients in the formulation of such vulnerability.

A second cognitive theory of the onset of depression is the helplessness/hopelessness model. As proposed by Abramson, Seligman, and Teasdale (1978), the helplessness model conceptualises cognitive vulnerability as one's tendency to make particular attributions for life events. Specifically, this theory suggests that individuals attributing negative life events to causes that are global, stable, and internal are particularly prone to depression. Further, the hopelessness theory, a reformulation of helplessness theory (Abramsom et al., 1989) follows that in the presence of negative but not positive life events, depressogenic attributional style interacts with these negative events, and acts as a diathesis to increase the likelihood of experiencing depressive symptoms (Abela, 2001; Abramson et al., 1989; Hankin, Abramson, Miller, \& Haeffel, 2004). This theory focuses on three depressogenic inferential styles or attributional tendencies: the assignment of global and stable attributions for the cause of negative events; the perception of negative consequences for stressors; and 
the tendency to hold negative views of the self in the presence of negative events. Tests of the helplessness and hopelessness theories of depression have been promising (Hankin, 2008; Hankin, Abramson, \& Stiler, 2001; Haeffel et al., 2008; Ingram et al., 1998).

Finally, self-esteem, or one's perceived overall value of the self, is a cognitive vulnerability that cuts across a wide number of theoretical models of depression (Abramson et al., 1989; Beck, 1967; Brown \& Harris, 1978). However, the influence of negative self-cognitions on depression differs from the manner proposed by models of overall low self-esteem (Brown \& Harris, 1978), the activation of negative self schema (Beck, 1967), labile self-esteem (Roberts \& Gotlib, 1997), or negative inferences of the self following stressors (Abramson et al., 1989). Although the importance of self-concept is clearly seen across many theoretical frameworks, it is an inconsistent predictor of changes in depression in prospective studies (Roberts \& Monroe, 1994).

\section{Integrated models of vulnerability}

Multiple models of cognitive vulnerability to depression have thus been proposed, each of which posits that maladaptive beliefs or styles of thinking predispose individuals to developing depression, particularly in the context of stressful life events. Notably, what differentiates these theories are the specific cognitive vulnerabilities emphasised by each model. Regardless of these comparisons, in each of the corresponding literatures, a significant number of inconsistent results have troubled researchers. A number of possible explanations for these inconsistencies have been proposed, including the use of older and less reliable measures (Haeffel et al., 2008), state-dependence of negative cognition (Miranda \& Persons, 1990), the requirement of mood primes (Teasdale, 1988), the need to address possible subtypes of depression such as hopelessness depression (Abramson et al., 1989), and the need for more idiographic assessments of both stress and cognition (Abela, Aydin, \& Auerbach, 2006).

Abela and Sarin (2002) were particularly interested in the inconsistent results observed in tests of the hopelessness theory among adolescents and proposed the weakest link hypothesis. They noted that the convention for testing aspects of attributional style involves treating the stable, global, and internal subscales separately or summing the stable and global (and sometimes internal) subscales. This latter convention treats all subscales as equal indices of a single general vulnerability factor. Yet Abela and Sarin proposed that these subscales need to be considered relative to each other, such that an individuals' best indicator of propensity to depression is the lowest (i.e., most maladaptive) of their three subscales. Just as the strength of a chain lies solely with its weakest link, individuals' overall propensity to depression may lie solely with their most vulnerable cognitive characteristic. Thus, indices of a person's average vulnerability or their greatest strength would be irrelevant in the prediction of depression. This weakest link hypothesis has performed well in tests of the hopelessness theory (Abela et al., 2006; Abela \& McGirr, 2007; Abela, McGirr, \& Skitch, 2007; Abela \& Sarin, 2002), though this was not independently replicated in a recent study of Spanish adolescents (Calvete, Villardon, Estevez, 2008). 
In a recent study, Haeffel (2010) investigated the predictive utility of the weakest link hypothesis to predict changes in depressive symptoms in a sample of 251 college students. This study is unique in that it is the first test of the weakest link model that examined its incremental validity, after the effects of "traditional" attributional style (scored as the average of the subscales) were controlled. Though there was a significant interaction of stress with the weakest link composite, this effect was no longer significant after partialling out the effects of the traditional composite. Importantly, Haeffel found that the weakest link composite score correlated .93 with the traditional composite scoring. As such, he concluded that the weakest link composite may not represent a unique conceptualisation of cognitive vulnerability over the traditional (average of subscales) composite. The author also noted that he replicated this pattern of results in two other unpublished investigations. On the basis of wider breadth of assessment and increased measurement reliability, Haeffel suggested that the traditional method should take precedence over the weakest link.

One of the concerns noted in Haeffel (2010) was the very high correlation between the weakest link composite and the traditional additive composite. A potential explanation for this high correlation among composites is that the individual indices used to form the weakest link composite may have been too conceptually and statistically interrelated. As noted in Haeffel's (2010) paper (p. 93) "this [weakest link] scoring approach assumes that the three vulnerability components are relatively independent of one another". Returning to Abela's concept of a link of connected chains, if every chain were identical and would break under the exact same strain, there would be no value in searching for the weakest component. Thus, the value of a weakest link strategy is optimised when integrating multiple valid and orthogonal mechanisms through which depression may occur. However, rarely are multiple indices either completely redundant or totally orthogonal; thus the potential utility of the weakest link is influenced by the relative association among the indices, with lower associations being preferable. As such, this type of idiographic approach may lack utility in combining indices from one theoretical perspective (as found in Haeffel, 2010) but have promise in integrating information from multiple theories and more distinct vulnerabilities.

Morris et al. (2008) extended the logic of the weakest link hypothesis from hopelessness theory alone to cognitive vulnerability in general. Using measures of attributional style, selfworth, and hopelessness, scores were first standardised, and then individuals' weakest link scores were computed based on the score of the most maladaptive scale for each person. This application of the weakest link strategy thus attempted to integrate the risks for depression based on multiple theoretical backgrounds. The resulting weakest link composite in this study did not show the excessively high correlation observed in the Haeffel (2010) paper. This new approach is reflective of the concept of equifinality, wherein different vulnerabilities may represent distinct alternate paths to the same result of depression. The assumption of equifinality complicates the prediction of depression; if there are qualitatively different pathways that individuals may take to arrive at depression, how can we best quantify a person's overall risk for disorder? The weakest link approach may be quite useful in this regard as it assumes multiple distinct vulnerabilities, one common outcome, and that the measurement of overall risk is best reflected by the pathway to disorder that is most salient for each person. 
Morris and colleagues (2008) extended the logic of this type of idiographic approach beyond the assessment of vulnerability by also examining resilience. A keystone model was investigated in which an individual's most adaptive cognitive style is thought to determine one's susceptibility to depression. Using a sample of young adolescents, Morris and colleagues found support for individual, additive, weakest link and keystone models. A significant interaction of the weakest link model with stress and gender was found to predict depressive symptoms, suggesting that the theory proposed by Abela and Sarin (2002) can be extended as a useful framework for studying cognitive vulnerability in general, rather than being limited to attributional styles. Furthermore, main effects also supported the keystone model; individuals' most adaptive cognitive scores were protective against depression. Finally, an additive model consisting of the mean of all cognitive indices also interacted with stress in predicting depression.

Importantly, Morris and colleagues (2008) did not directly examine the incremental utility of the weakest link and keystone models over the additive (mean score) model. Examination of the zero-order correlations among these three composites suggested that, in contrast to the results of Haeffel (2010), these three composites are empirically distinct. Thus, the resulting composites did not show the level of redundancy seen in Haeffel's investigation. This suggests that the weakest link and keystone approaches may be more useful when examining the influence of more diffuse indicators of vulnerability. To our knowledge, the incremental validity of the weakest link and keystone approaches has not been investigated as applied to more distinct indicators of risk.

The aim of the present study was twofold: (1) To compare the utility and incremental validity of individual negative cognitive indices drawn from multiple theoretical perspectives; and (2) to compare the additive, weakest link, and keystone composites as predictors of depressive symptoms in a longitudinal study of university students. Thus, we sought to further the work of Morris and colleagues (2008) by not only examining multiple theoretically distinct depression vulnerabilities using weakest link and keystone approaches, but also by testing the incremental validity of these risk composites over the traditional additive approach.

Three theoretical frameworks were utilised in this study: Beck's cognitive theory, the helplessness/hopelessness theories, and self-esteem theories of depression. From Beck's theory, we examined the Dysfunctional Attitudes Scale (DAS) and the Cognitive Triad Inventory (CTI). From the helplessness/hopelessness theories, we examined the Attributional Style Questionnaire (ASQ), and from the self-esteem perspective, we utilised the Rosenberg Self-Esteem Questionnaire (RSE). These measures are also highly similar to the various cognitive constructs assessed in the Morris and colleagues' (2008) study of vulnerability among adolescents, which examined adolescent-specific measures from the hopelessness and self-esteem perspectives on vulnerability to depression. The present study used corresponding adult measures of these constructs and also extended the focus to include indices from Beck's cognitive theory of depression. This latter extension is notable due to the long history of contrasting the Beck and hopelessness theories of depression (Hankin et al., 2004; Lewinsohn, Joiner, \& Rohde, 2001). Though the selected constructs play an arguably different causal role in various cognitive theories, they were selected 
because each assesses a type of belief or cognitive content that has been demonstrated to be a vulnerability to depression in multiple studies. These vulnerabilities and their resulting composites were used as predictors of depression in a six-week longitudinal study.

\section{METHOD}

\section{Participants}

Participants for this investigation were recruited from introductory psychology classes at Kent State and Vanderbilt Universities. Students signed up to participate in a study of "College Students' Thoughts and Feelings" involving a one-time completion of questionnaires in the laboratory. A total of 621 (391 female) students completed questionnaires in the parent study to complete a course requirement. Additionally, 390 of these participants gave permission for study staff to contact them for potential follow-up. Attempts were made to re-contact these participants, to recruit them for a separate follow-up investigation. E-mails were sent to 390 of the original participants who provided contact information, and 140 (108 female) individuals of the original sample were successfully recruited to complete a second round of questionnaires, six weeks following their participation in the first study. Participants were compensated $\$ 15$ for participation in the follow-up.

\section{Measures}

Beck Depression Inventory-II (BDI-II; Beck, Steer, \& Brown, 1996)—The BDI is a 21-item questionnaire used at times 1 and 2 to assess depressive symptoms on a 4-point scale. In the present study, BDI-II scores were $z$-scored and averaged with the CES-D to create a composite measure of depressive symptoms. This measure is widely used and has shown excellent internal consistency and test retest reliability. In the present study, the coefficient alpha was .87 .

\section{Centers for Epidemiological Studies Depression Scale (CES-D; Radloff, 1977)}

-The CES-D is a 20-item self-report assessment of depressive symptomatology in the general population, focusing on affective problems associated with depression. Respondents were instructed to indicate how often they felt or behaved with regard to each statement (e.g., "I was bothered by things that usually don't bother me", "I felt sad", or "I felt hopeful about the future") this way in the past week on a 4-point scale from "rarely" to "most or all of the time". In the present study, the CES-D, given at times 1 and 2, was $z$-scored and averaged with the BDI-II to create a composite variable of depressive symptoms. The CESD has been shown to be psychometrically sound and demonstrates high test-retest reliability (Radloff, 1977). In the present sample, the coefficient alpha was .90.

Life Events Survey (LES; Sarason, Johnson, \& Siegel, 1978)—The LES assesses the number of stressful life events that occurred within the past six months with a checklist of 20 life events that cover a wide range of experiences that commonly occur to college students. The instructions were modified for this study, at both times 1 and 2, to report the number of events that have occurred in the past six weeks in order to provide coverage for the interval between baseline and follow-up data collections. 
The Cognitive Triad Inventory (CTI; Beckham, Leber, Watkins, Boyer, \& Cook, 1986)-The CTI was given at times 1 and 2, it assesses Beck's cognitive triad of negative perceptions of the self, world and future. Representative items include "I can do a lot of things well" (view of self), "The world is a very hostile place" (world view), and "There is nothing to look forward to in the years ahead" (view of future). To ease interpretation across scales, the CTI was scored in reverse of convention, with high scores indicating more negative perceptions of self, world, and future. In the present sample, coefficient alpha was . 91.

\section{Rosenberg Self-Esteem Questionnaire (RSE; Rosenberg, 1979)—The RSE}

measures participants' global self-worth using a 10-item scale on which participants respond to general self-concept statements (e.g., "At times, I think I am no good at all" and "On the whole, I am satisfied with myself"). The RSE demonstrates good test-retest reliability ( $r=$ 85 to .88; Rosenberg, 1979). In the present sample, coefficient alpha was .90. RSE scores were scored in the reverse of convention, such that higher scores indicate lower self-esteem. The RSE was given at times 1 and 2.

Dysfunctional Attitude Scale (DAS; Weissman \& Beck, 1978)—The DAS is a 40item scale, given at times 1 and 2, that assesses rigid and perfectionistic beliefs, considered vulnerabilities in Beck's theory of depression (e.g., "I cannot be happy unless most people I know admire me" and "People will probably think less of me if I make a mistake"). In the present study, the coefficient alpha was .89 .

Attributional Style Questionnaire (ASQ; Peterson et al., 1982)—The ASQ, ${ }^{1}$ given at times 1 and 2, assesses attributions or explanations for six hypothetical events.

Respondents are asked to report the likelihood that each event was caused by the self (internal), would have lasting negative consequences (stable), and would have negative implications for the other domains of the respondent's life (global). Scores were summed to create a composite with higher scores indicating more negative attributional style. In the present study, the coefficient alpha was .64.

Cognitive composites-The additive, weakest link and keystone hypotheses were computed from the RSE, CTI, DAS, and ASQ in the following ways. First, $z$-scores were computed for each of these measures separately, thus placing them on the same metric. Scores thus had a mean of zero, standard deviation of one, and higher scores reflect greater theoretical vulnerability. To compute the additive composite, the mean of these four $z$-scores were computed. Note that the mean and sum are perfectly correlated and, except for scaling, the distinction between them is arbitrary. To compute the weakest link score, the highest (most maladaptive) score from these found indices was used. To compute the keystone score, the lowest (most adaptive) score was used.

${ }^{1}$ The ASQ was used in this study rather than the more reliable Cognitive Style Questionnaire because the latter is very lengthy and requires over 30 minutes to complete (see Haeffel et al., 2008). 


\section{RESULTS}

\section{Preliminary analyses}

Our first set of analyses explored the prediction of successful recruitment for the follow-up investigation from participants' baseline data. Gender was statistically associated with follow-up recruitment, such that males were less likely to participate at time $2, t(265)=$ $-4.7, p<.001$. Attrition was not predicted by time 1 age, depression, or measures of negative cognition or stress. Due to these results, gender was used as a covariate in subsequent analyses.

\section{Descriptive analyses}

Table 1 displays means, standard deviations, and correlations among study variables. Individual cognitive indices at time 1 were standardised as $z$-scores, after reverse-coding of the CTI and RSE were performed to create consistency in scores such that higher scores are indicative of more negative cognition. Additive scores were computed by averaging ${ }^{2}$ the $z$ scores of each participant's individual cognitive indices (CTI, DAS, RSE, ASQ). Weakest link scores were computed by selecting the highest (most negative) $z$-score of each participant's individual cognitive indices. Keystone scores were computed by selecting the lowest (least negative) $z$-score of each participant's individual cognitive indices. The weakest link scores were contributed by the RSE (21\%), CTI (22\%), DAS (22\%), and ASQ (35\%). Keystone scores were contributed by the RSE (19\%), CTI (21\%), DAS (27\%), and ASQ (33\%).

\section{Analyses of individual indices}

Before examining the integrative models, we explored the predictive value of the individual measures of negative cognition (i.e., CTI, DAS, RSE, ASQ) in separate models. These models included gender, time 1 depression, and the LES at times 1 and 2 as covariates in the first block, and predicted time 2 depression. The second block included the main effects of cognition. The final block added the interaction of cognition and stress. The ASQ was not a significant predictor of depression in either a main effect $\left(\beta=0.058, \Delta R^{2}=.003, p=.48\right.$ ) or in an interaction with stress $\left(\beta=0.126, \Delta R^{2}=.015, p=.12\right)$. The CTI showed a main effect ( $\left.\beta=0.301, \Delta R^{2}=.046, p<.01\right)$, but no stress interaction $\left(\beta=-0.005, \Delta R^{2}=.000, p=.95\right)$. The RSE showed a marginal main effect $\left(\beta=0.164, \Delta R^{2}=.018, p=.09\right)$, but no stress interaction $\left(\beta=-0.072, \Delta R^{2}=.003, p=.45\right)$. Finally, the DAS did not show a main effect ( $\beta$ $\left.=0.136, \Delta R^{2}=.013, p=.15\right)$ or stress interaction $\left(\beta=0.024, \Delta R^{2}=.000, p=.79\right)$. In order to examine potential unique effects, we explored a regression equation that included all main effects in the second step, and none were significant ( $p s>.13)$. The addition of all cognition $\times$ stress interaction terms also yielded non-significant results for all indices $(p \mathrm{~s}>.35)$.

\section{Integrative effects}

Next, we used multiple regression analyses to examine the association of each of the three proposed integrative models measured at time 1 as predictors of depression at time 2 ,

\footnotetext{
${ }^{2}$ When the number of indices being combined is constant, as it is in this paper, the average and sum of scores are perfectly correlated and statistically interchangeable.
} 
controlling for depression at time 1. Each composite was examined in a separate regression. We entered previous depression and the LES at times 1 and 2 in the first block of predictors, each integrative index of vulnerability in the second block and the vulnerability $\times$ stress interaction in the third and final block (see Table 2). The additive model demonstrated a significant main effect in the prediction of depression, accounting for approximately $5 \%$ of the variance. However, the additive composite did not interact with life stress. Similarly, the weakest link model showed a main effect that accounted for roughly $7 \%$ of the variance in depression and this composite also did not interact with stress. Finally, the main effect of the keystone model accounted for about $3 \%$ of the variance and did not interact with life stress.

\section{Incremental effects}

As the weakest link and keystone models are more complex (and less parsimonious) than an additive model, we tested whether these two models had incremental validity over the additive model in two sets of hierarchical regression models. In the first model, after controlling for the effects of gender, time 1 and 2 stress, time 1 depression and the additive composite, the weakest link composite was added. The weakest link demonstrated a significant incremental effect over the additive composite $\left(\beta=0.30, \Delta R^{2}=.023, p<.05\right)$. Due to the high correlation between these composite variables, we also examined the Variance Inflation Factor (VIF or 1/Tolerance) and the Condition Index for evidence that our results may have been unduly influenced by multicollinearity. Scores exceeding 30 on the Condition Index for the overall model or 10 on the VIF for any individual predictor would indicate cause for concern (see Cohen, Cohen, West, \& Aiken, 2003, pp. 422-425). For this first model, the Condition Index was 11.74 and the highest VIF was 4.71. In the second hierarchical regression, the keystone model was not a statistically significant predictor of depression after controlling for the effects of time 1 depression and the additive model $(\beta=$ $\left.-0.06, \Delta R^{2}=.001, p=.73\right)$. In this model, the Condition Index was 11.59 and the highest VIF was 5.97.

Finally, in a series of exploratory analyses, we also examined the unique effects of the integrative composites and the individual vulnerabilities (i.e., ASQ, DAS, CTI, RSE) when placed in the same models. For these analyses, regression models were specified in which the first block included prior depression and the second block included one individual vulnerability and one integrative composite. Thus, twelve separate models ( 3 composites $\times 4$ vulnerabilities) were run to examine how they performed in a simultaneous regression. In every analysis, across every integrative composite, the individual vulnerability indices were non-significant ( $p \mathrm{~s}>.11)$. A single exception emerged such that the CTI had a significant effect $(p=.026)$ after the keystone composite was included.

When compared to the individual vulnerabilities in separate regression models, the keystone composite was never a significant predictor of changes in depression ( $p \mathrm{~s}>.07)$. The additive composite showed significant incremental value over the DAS $\left(\beta=0.323, \Delta R^{2}=.03, p<\right.$. $05)$, the $\operatorname{RSE}\left(\beta=0.392, \Delta R^{2}=.05, p<.05\right)$, and the ASQ $\left(\beta=0.328, \Delta R^{2}=.04, p<.01\right)$, but not the CTI $\left(\beta=0.179, \Delta R^{2}=.02, p=.19\right)$. Finally, the weakest link composite remained significant regardless of which individual vulnerability was controlled; after controlling for the ASQ $\left(\beta=0.338, \Delta R^{2}=.07, p<.001\right)$, the CTI $\left(\beta=0.263, \Delta R^{2}=.04, p^{<.01}\right)$, the DAS ( $\beta$ 
$\left.=0.326, \Delta R^{2}=.05, p<.01\right)$, and the $\operatorname{RSE}\left(\beta=0.256, \Delta R^{2}=.03, p<.05\right)$. Multicollinearity

diagnostics in these models were not indicative of problematic results (all Condition Indices B11.3 and VIFs B6.0).

\section{DISCUSSION}

This investigation of multiple cognitive vulnerabilities to depression specifically focused on the utility of two recent conceptualisations of cognitive risk, the weakest link and keystone models. A range of vulnerability factors, cutting across multiple theoretical backgrounds, were measured in this study as predictors of changes in depression. Each of these four individual vulnerabilities was positively associated with changes in depression, though this effect was not significant for two, significant for one, and marginal for another. However, no interactions with life stress were observed for these predictors.

When we separately examined methods for integrating various indices of cognitive risk, we found support for all three conceptualisations (additive, weakest link, and keystone models) of risk in the prediction of depression. Similar to the individual cognitive predictors, the results were consistent with main effects rather than interactions with life stress. Importantly, we then examined the incremental utility of the weakest link and keystone approaches. We considered the additive composite to have precedence as it makes the parsimonious assumption that the individual measures are reflective of general cognitive risk. The validity of a general cognitive risk factor for depression is currently a matter of debate, with some studies suggesting that different cognitive measures represent distinct constructs (e.g., Hankin, Lakdawalla, Carter, Abela, \& Adams, 2007), though others suggest that they account for the same variance in depression (e.g., Hankin et al., 2004).

The keystone composite did not perform well in this study, in two important respects. First, though this index was significantly predictive of changes in depression, it did not demonstrate any incremental predictive value over the additive composite. Second, it also did not demonstrate incremental utility over any individual vulnerability measure. This suggests that cognitive risk for depression does not uniquely vary as a function of a person's lowest vulnerability. Morris and colleagues (2008) previously found support for the keystone hypothesis in the form of a main effect (but not in an interaction with stress) similar to our results. However, this prior study did not directly test the keystone hypothesis after first controlling for the additive model or effects of the individual predictors. We caution against an interpretation of our results as suggesting that resiliency is not relevant in the prediction of depression. Though keystone scores were originally presented as measures of resiliency (Morris et al., 2008), it is more accurate to refer to these scores as indices of one's lowest vulnerability, which is conceptually distinct from resiliency. In contrast, the weakest link composite did show promise in our results. After controlling for the additive composite, the weakest link offered significant incremental utility in predicting changes in depression. Further, the weakest link composite remained a significant predictor of depression after controlling for the individual cognitive vulnerabilities. Importantly, the additive model did not perform as well in the same tests; it was no longer a significant predictor once the RSE and CTI were controlled but was still significant when the ASQ and DAS were partialled out. Thus, the weakest link composite was predictive of depression 
alone, after controlling for the additive composite and after controlling for each of the individual predictors. As such, this investigation supports the use of the weakest link method of integrating multiple measures of cognitive beliefs.

However, our findings need to be considered in light of the high correlations among the three composite scores. Though the multicollinearity diagnostics of the regression models did not reveal a statistical problem, conceptual concerns remain. The weakest link composite accounted for an additional $2 \%$ of the variance in depression after controlling for the additive composite. Though statistically significant, it is uncertain whether this would translate into a clinically useful increment in predicting onsets of depression. Thus, the weakest link approach performed well in this investigation, though due to the great overlap with the simpler additive model, we believe that at present it would be premature to provide an unqualified recommendation for its routine use. As this is only the second investigation to apply the weakest link approach to integrate cognitive risk factors from different theories (see also Morris et al., 2008), additional research is needed, particularly with clinical samples.

As noted, Haeffel (2010) recently conducted an analogous investigation of different models of cognitive risk in the prediction of depression. He investigated these models in the context of the hopelessness theory (Abramson et al., 1989), and focused on different methods of scoring the Cognitive Style Questionnaire (CSQ; Haeffel et al., 2008). Haeffel found support for the "traditional" method of scoring the CSQ, which is analogous to the additive model of the current study. Little support, however, was found for other conceptualisations, including the weakest link. Although our methods and results differed in that we found support for the weakest link strategy, it is important to note that we applied the weakest link approach in a different way. As noted in the introduction, we utilised the multitheoretical approach of Morris and colleagues (2008) in an attempt to investigate whether the weakest link could demonstrate incremental validity over the additive approach when measures of conceptually distinct vulnerabilities are utilised. We selected multiple measures of cognitive beliefs from various theoretical perspectives, though we acknowledge that there are many other possible measures that could be examined in a similar application. As this type of idiographic approach is still quite novel, future studies are needed that develop more theoretically derived criteria for the selection of specific measures from which to form weakest link composites.

Despite the large number of significant predictors of change in depression during the study, cognitive risk $\times$ life stress interactions were not observed. This is interesting considering that most the theories examined in this study are diathesis-stress theories. We believe that there are a few possible explanations for the lack of interactive effects. First, the study period was relatively brief and thus we may have not captured a sufficient number of stressors to demonstrate these effects. Second, we used a self-report checklist of life stress, which may lack the sensitivity of interview-based assessments as exemplified by the Life Events and Difficulties Schedule (Brown \& Harris, 1978). Third, the sample may have been under a generally high level of life stress of a type not easily captured by checklists. Participants were university students taking freshman level classes. The transition into college is a period of time of profound change and stress (Towbes \& Cohen, 1996). To the extent that a given 
sample is under high stress, one would actually anticipate stronger main effects (the vulnerabilities would be easily manifest) and weaker interactive effects (as there is a lack of individuals experiencing little-to-no stress). Finally, we also note the well-documented statistical issues in testing for interactions in survey research (McClelland \& Judd, 1993). Statistical power for detecting interactions in such designs tends to be much lower than power to detect main effects.

The present study did have some limitations that could be addressed in future research. First, self-report measures of study variables were universally used. Later studies could benefit from the inclusion of interview-based assessments of depression and life stress in particular. Additionally, various information-processing and psychophysiological methodologies have been developed to measure cognitive risk factors which could be utilised. Further, though the sample size of this longitudinal study was large, it represented a modest percentage of individuals from the initial parent cross-sectional study. This level of participation is actually quite good, given that participants were not initially recruited for a longitudinal study and only a subset volunteered contact information for potential follow-up. However, it is possible that the individuals who participated in the follow-up differed from the sample as a whole in unknown ways. Further, we conducted a number of exploratory analyses to examine the relative influence of individual predictors, but did so without correction for error inflation. This was done to maximise sensitivity to significant effects, though it does suggest that the results of follow-up tests should be interpreted with caution. Finally, the sample of university students may limit the generalisability of findings. Yet, rates of depressive, substance, and anxiety disorders are actually remarkably high among college students, and are not significantly different from rates observed among non-college students (Blanco et al., 2008). Although this suggests these findings would likely generalise to a community sample, it will still be important to examine these phenomena in a more diverse population.

Our findings have potential clinical implications. Every client has their own constellation of cognitive vulnerabilities, and therapists frequently have to decide which aspects to target for change. The additive model performed well in this study, which suggests that clinicians could effectively pursue change by focusing on any aspect of cognitive vulnerability.

However, the incremental utility of the weakest link model suggests that a multidimensional assessment of cognitive risk prior to treatment would also have merit. This would allow therapists to tailor treatment to specifically target each client's greatest vulnerability, which the weakest link approach suggests would be the most effective way to produce symptomatic improvement. Yet, as the weakest link model is still a novel approach to understanding cognitive vulnerability, future aetiological and clinical studies are needed to validate such predictions.

\section{REFERENCES}

Abela JRZ. The hopelessness theory of depression: A test of the diathesis-stress and causal mediation components in third and seventh grade children. Journal of Abnormal Child Psychology. 2001; 29:241-254. [PubMed: 11411786] 
Abela JRZ, Aydin C, Auerbach RP. Operationalizing the "vulnerability" and "stress" components of the hopelessness theory of depression: A multi-wave longitudinal study. Behavior Research and Therapy. 2006; 22:1565-1583.

Abela JRZ, McGirr A. Operationalizing cognitive vulnerability and stress from the perspective of the hopelessness theory: A multi-wave longitudinal study of children of affectively ill parents. British Journal of Clinical Psychology. 2007; 46:377-395. [PubMed: 17535532]

Abela JRZ, McGirr A, Skitch SA. Depressogenic inferential styles, negative events, and depressive symptoms in youth: An attempt to reconcile past inconsistent findings. Behaviour Research and Therapy. 2007; 45:2397-2406. [PubMed: 17475210]

Abela JRZ, Sarin S. Cognitive vulnerability to hopelessness depression: A chain is only as strong as its weakest link. Cognitive Therapy and Research. 2002; 26:811-829.

Abramson LY, Metalsky GI, Alloy LB. Hopelessness depression. Psychological Review. 1989; 96:358-372.

Abramson LY, Seligman ME, Teasdale JD. Learned helplessness in humans: Critique and reformulation. Journal of Abnormal Psychology. 1978; 87:49-74. [PubMed: 649856]

Beck, AT. Depression: Clinical, experimental, and theoretical aspects. Harper \& Row; New York, NY: 1967.

Beck AT. The current state of cognitive therapy: A 40-year retrospective. Archives of General Psychiatry. 2005; 62:953-959. [PubMed: 16143727]

Beck, AT.; Steer, RA.; Brown, GK. Manual for the Beck Depression Inventory-II. Psychological Corporation; San Antonio, TX: 1996.

Beckham EE, Leber WR, Watkins JT, Boyer JL, Cook JB. Development of an instrument to measure Beck's cognitive triad: The Cognitive Triad Inventory. Journal of Consulting and Clinical Psychology. 1986; 54(4):566-567. [PubMed: 3745613]

Blanco C, Okuda M, Wright C, Hasin DS, Grant BF, Liu SM, et al. Mental health of college students and their non-college attending peers. Archives of General Psychiatry. 2008; 65:1429-1437. [PubMed: 19047530]

Brown, GW.; Harris, TO. Social origins of depression: A study of psychiatric disorder in women. Free Press; New York, NY: 1978.

Calvete E, Vallardon L, Estevez A. Attributional style and depressive symptoms in adolescents: An examination of the role of various indicators of cognitive vulnerability. Behaviour Research and Therapy. 2008; 46:944-953. [PubMed: 18533131]

Ciesla JA, Roberts JE. Rumination, negative cognition, and their interactive effects on depressed mood. Emotion. 2007; 7:555-565. [PubMed: 17683212]

Cohen, J.; Cohen, P.; West, SG.; Aiken, LS. Applied multiple regression/correlation analysis for the behavioral sciences. 3rd ed.. Lawrence Erlbaum Associates, Inc.; Hillsdale, NJ: 2003.

Haeffel GJ. Cognitive vulnerability to depressive symptoms in college students: A comparison of traditional, weakest-link, and flexibility operationalizations. Cognitive Therapy and Research. 2010; 34:92-98.

Haeffel GJ, Gibb BE, Metalsky GI, Alloy LB, Abramson LY, Hankin BL, et al. Measuring cognitive vulnerability to depression: Development and validation of the cognitive style questionnaire. Clinical Psychology Review. 2008; 28:824-836. [PubMed: 18234405]

Hankin BL. Cognitive vulnerability-stress model of depression during adolescence: Investigating depressive symptom specificity in a multi-wave prospective study. Journal of Abnormal Child Psychology. 2008; 36:999-1014. [PubMed: 18437551]

Hankin BL, Abramson LY, Miller N, Haeffel GJ. Cognitive vulnerability-stress theories of depression: Examining affective specificity in the prediction of depression versus anxiety in 3 prospective studies. Cognitive Therapy and Research. 2004; 28:309-345.

Hankin BL, Abramson LY, Stiler M. A prospective test of the hopelessness theory of depression in adolescence. Cognitive Therapy and Research. 2001; 25:607-632.

Hankin BL, Lakdawalla Z, Carter IL, Abela JRZ, Adams P. Are neuroticism, cognitive vulnerabilities and self-esteem overlapping or distinct risks for depression? Evidence from confirmatory factor analyses. Journal of Social and Clinical Psychology. 2007; 26:29-63. 
Ingram, RE.; Miranda, J.; Segal, ZV. Cognitive vulnerability to depression. Guilford Press; New York, NY: 1998.

Lewinsohn PM, Joiner TE, Rohde P. Evaluation of cognitive diathesis-stress models in predicting major depressive disorder in adolescents. Journal of Abnormal Psychology. 2001; 110:203-215. [PubMed: 11368074]

McClelland GH, Judd CM. Statistical difficulties of detecting interactions and moderator effects. Psychological Bulletin. 1993; 114:376-390. [PubMed: 8416037]

Miranda J, Persons JB. Endorsement of dysfunctional beliefs depends on current mood state. Journal of Abnormal Psychology. 1990; 99:237-241. [PubMed: 2212273]

Morris MC, Ciesla JA, Garber J. A prospective study of the cognitive-stress model of depressive symptoms in adolescents. Journal of Abnormal Psychology. 2008; 117:719-734. [PubMed: 19025221]

Peterson C, Semmel A, von Baeyer C, Abramson LY, Metalsky GI, Seligman ME. The attributional style questionnaire. Cognitive Therapy and Research. 1982; 6:287-299.

Radloff LS. The CES-D scale: A self-report depression scale for research in the general population. Applied Psychological Measurement. 1977; 1(3):385-401.

Roberts JE, Gotlib IH. Temporal variability in global self-esteem and specific self-evaluation as prospective predictors of emotional distress: Specificity in predictors and outcome. Journal of Abnormal Psychology. 1997; 106:521-529. [PubMed: 9358682]

Roberts JE, Monroe SM. A multidimensional model of self-esteem in depression. Clinical Psychology Review. 1994; 14:161-181.

Rosenberg, M. Conceiving the self. Basic Books; New York, NY: 1979.

Sarason IG, Johnson JH, Siegel JM. Assessing the impact of life changes: Development of the Life Experiences Survey. Journal of Consulting and Clinical Psychology. 1978; 46:932-946. [PubMed: 701572]

Teasdale JD. Cognitive vulnerability to persistent depression. Cognition and Emotion. 1988; 2:247274.

Towbes LC, Cohen LH. Chronic stress in the lives of college students: Scale development and prospective prediction of distress. Journal of Youth and Adolescence. 1996; 25:199-217.

Weissman, AN.; Beck, AT. Development and the validation of the Dysfunctional Attitude Scale; Paper presented at the meeting of the Association for the Advancement of Behavior Therapy; Chicago. 1978; Nov. 


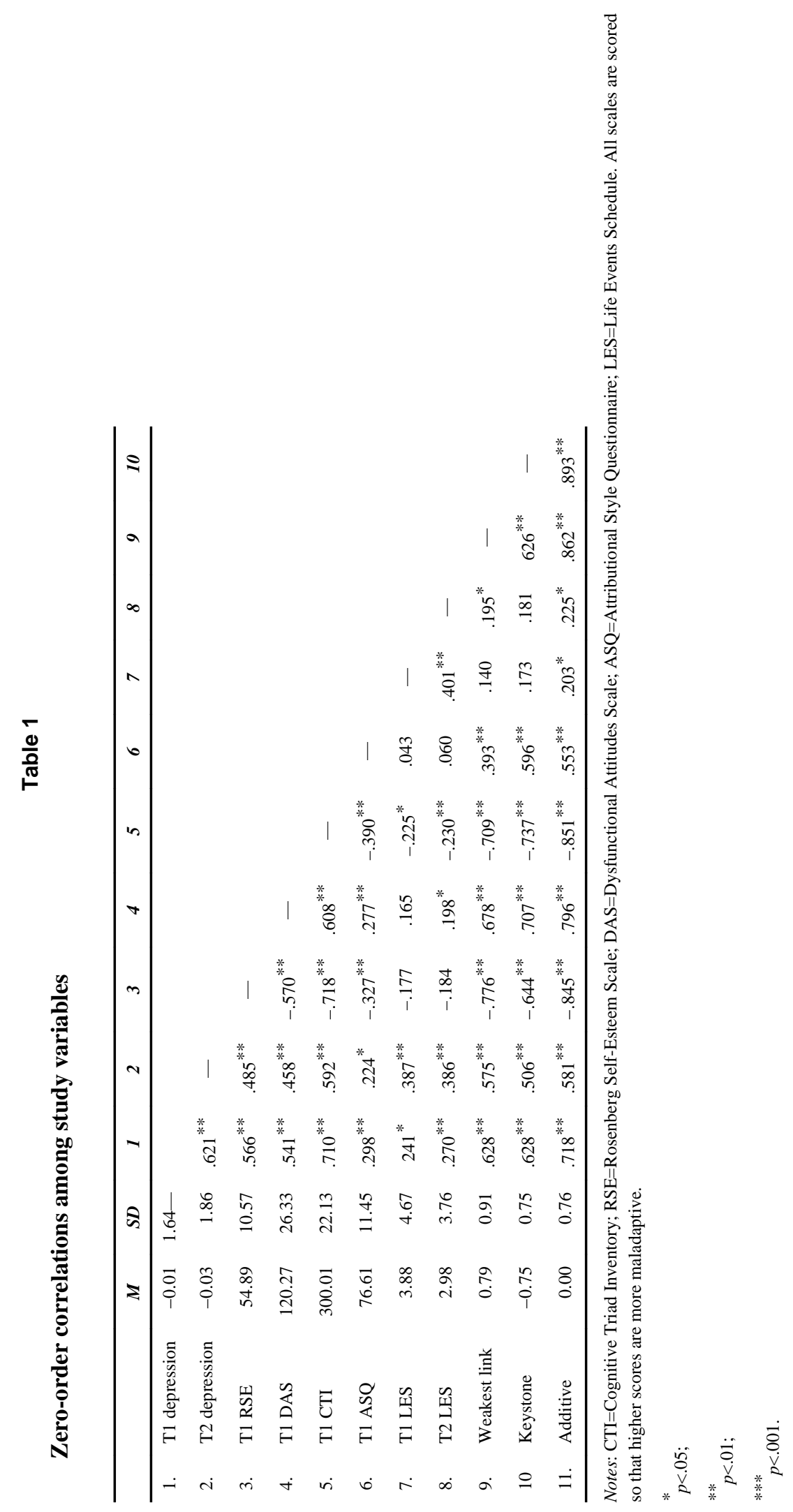


